\title{
Bioassay, In Vivo
}

National Cancer Institute

\section{Source}

National Cancer Institute. Bioassay, In Vivo. NCI Thesaurus. Code C18641.

A laboratory test or analysis of the biological properties or activities of a substance performed by testing its effect on an organism. 